Article

\title{
Is the Hawking Quasilocal Energy “Newtonian”?
}

\section{Valerio Faraoni}

Physics Department and STAR Research Cluster, Bishop's University, 2600 College Street, Sherbrooke, Québec, Canada J1M 1Z7; E-Mail: vfaraoni@ubishops.ca; Tel.: +1-819-822-9600 (ext. 2490); Fax: +1-819-822-9611

Academic Editor: Shin'ichi Nojiri

Received: 20 October 2015 / Accepted: 28 October 2015 / Published: 5 November 2015

\begin{abstract}
The Misner-Sharp-Hernandez mass defined in general relativity and in spherical symmetry has been recognized as having a Newtonian character in previous literature. In order to better understand this feature we relax spherical symmetry and we study the generalization of the Misner-Sharp-Hernandez mass to general spacetimes, i.e., the Hawking quasilocal mass. The latter is decomposed into a matter contribution and a contribution coming solely from the Weyl tensor. The Weyl tensor is then decomposed into an electric part (which has a Newtonian counterpart) and a magnetic one (which does not), which further splits the quasilocal mass into "Newtonian" and "non-Newtonian" parts. Only the electric (Newtonian) part contributes to the quasilocal mass.
\end{abstract}

Keywords: quasilocal energy; Hawking mass; classical general relativity

\section{Introduction}

The concept of mass of a gravitating system in general relativity, especially a non-isolated one, has been the subject of much research. The equivalence principle forbids the introduction of an energy density for the gravitational field because, locally, one can eliminate this field. The next best thing seems to be introducing a quasilocal energy, and this avenue has been pursued for a long time with the introduction of many definitions of quasilocal energy (see [1] for a review). Some emphasis seems to be given, in recent literature, to the Hawking and Hayward constructs. It is fair to say that a definitive prescription which is appropriate for all problems in relativity does not yet exist, with various definitions being applied to different problems and for different purposes. This situation is far from ideal and, overall, quasilocal energies remain rather abstract and formal concepts, at least for non-asymptotically flat geometries. It is only recently that the Hawking-Hayward quasilocal construct has been applied 
to more "practical" problems in cosmology, such as the Newtonian simulations of large scale structure formation [2] and the old problem [3-21] of the turnaround radius of the largest bound structures in the universe [22].

The problem of deciding once and for all what is the "mass of a gravitating system" in general relativity is far from being solved, and the physical understanding of the various quasilocal definitions is the first step in this direction. The present manuscript contributes by examining the "Newtonian" character of the Hawking mass. It has been pointed out [23] that, in a spherically symmetric spacetime, the behaviour of timelike geodesics in general relativity discriminates somehow between the Misner-Sharp-Hernandez [24,25] and the Brown-York [26] quasilocal energies. As seen from timelike geodesic observers, the Misner-Sharp-Hernandez mass (to which the Hawking mass reduces in spherical symmetry) plays the role of a Newtonian energy, while the Brown-York quasilocal energy plays the role of a relativistic energy [23]. This approach is intriguing, as it discloses from an unconventional but physical point of view, physical properties of these two quasilocal constructs which help understanding them better. Here, we want to go beyond the limitation of spherical symmetry of Reference [23] and we analyze the Hawking mass in general geometries. In order to make progress, one has to specify what is meant by "Newtonian" character of a quasilocal energy and we identify this property on the basis of the electric and magnetic decomposition of the Weyl tensor introduced long ago [27] and widely used in cosmology [28-35]. With this idea in mind, it is necessary to relate the Hawking mass $M_{\mathrm{H}}$ with the Weyl tensor. To this end, we first split the Hawking mass into two contributions, one due to matter and one to the "pure" gravitational field, i.e., to the Weyl tensor $C_{a b c d}$. If matter is described by a perfect fluid, the matter contribution to the quasilocal mass does not depend on the pressure.

As a second step, we perform the splitting of the Weyl tensor into its electric and magnetic parts in the gravitational (Weyl) contribution to $M_{\mathrm{H}}$ with the goal of identifying a part (coming from the electric part of $C_{a b c d}$ ) which has a Newtonian counterpart and another part (coming from the magnetic part of $C_{a b c d}$ ) which has no Newtonian counterpart. In so doing, we find that the gravitational contribution to $M_{\mathrm{H}}$ is due only to the electric part of $C_{a b c d}$ and is, in this sense, "Newtonian", while the magnetic part gives zero contribution, corroborating the result found by [23] in spherical symmetry (although the meaning of the adjective "Newtonian" is different in our context).

We use metric signature,$-+++ G$ is Newton's constant, round (resp., square) brackets around a pair of indices denote symmetrization (resp., antisymmetrization), units in which the speed of light is unity are used, and otherwise we follow the notation of Wald's textbook [36].

\section{Decomposing the Hawking Mass}

The Hawking-Hayward quasilocal mass contained by a 2 -surface $\mathcal{S}$ is defined in the following way [37,38]. Consider a spacetime $\left(M, g_{a b}\right)$ in general relativity and let $\mathcal{S}$ be a spacelike, embedded, compact, and orientable 2-surface in the spacetime manifold $M$. Let $h_{a b}$ and $\mathcal{R}^{(h)}$ be the 2-metric and Ricci scalar induced on $\mathcal{S}$ by the spacetime metric $g_{a b}$. Let $\mu$ be the volume 2-form on $\mathcal{S}$ and let $A$ be the area of $\mathcal{S}$. Consider the congruences of ingoing (-) and outgoing $(+)$ null geodesic emanating from the surface $\mathcal{S}$, and let $\theta_{( \pm)}$and $\sigma_{a b}^{( \pm)}$be the expansion scalars and the shear tensors of these congruences, 
respectively. Let $\omega^{a}$ be the projection onto $\mathcal{S}$ of the commutator of the null normal vectors to $\mathcal{S}$, i.e., the anoholonomicity [38]. The Hawking-Hayward quasilocal mass is [37,38]

$$
M_{\mathrm{HH}}=\frac{1}{8 \pi G} \sqrt{\frac{A}{16 \pi}} \int_{\mathcal{S}} \mu\left(\mathcal{R}^{(h)}+\theta_{(+)} \theta_{(-)}-\frac{1}{2} \sigma_{a b}^{(+)} \sigma_{(-)}^{a b}-2 \omega_{a} \omega^{a}\right)
$$

In spherical symmetry the Hawking-Hayward mass $M_{\mathrm{HH}}$ reduces to the Misner-Sharp-Hernandez mass [24,25] for a 2-sphere of symmetry and is a conserved Noether charge [39]. The Kodama vector (defined only in general relativity in the presence of spherical symmetry [40]) is used in place of a timelike Killing vector when none exists, and generates an energy current ("Kodama current") which, surprisingly, is conserved in the absence of timelike Killing vectors [40] (the "Kodama miracle" [41]). The Misner-Sharp-Hernandez mass is the conserved Noether charge corresponding to the conservation of the Kodama current [39].

If the term $-2 \omega_{a} \omega^{a}$ is dropped from Equation (1), $M_{\mathrm{HH}}$ reduces to the Hawking quasilocal prescription [37], which we denote by $M_{\mathrm{H}}$. The quantity $\omega_{a} \omega^{a}$ is gauge-dependent [1], which is a weakness of the construct (1), and we will drop it in the following, restricting ourselves to the Hawking mass $M_{\mathrm{H}}$.

We are now going to decompose $M_{\mathrm{H}}$ into two components, which can be identified as a contribution due to the mass-energy on the topological 2-sphere $\mathcal{S}$, and one due to the gravitational field.

We take advantage of the contracted Gauss equation [38]

$$
\mathcal{R}^{(h)}+\theta_{(+)} \theta_{(-)}-\frac{1}{2} \sigma_{a b}^{(+)} \sigma_{(-)}^{a b}=h^{a c} h^{b d} R_{a b c d}
$$

where $R_{a b c d}$ is the Riemann tensor, to compute the first three terms in the integral of Equation (1). The Riemann tensor splits into Ricci part and Weyl part [36]

$$
R_{a b c d}=C_{a b c d}+g_{a[c} R_{d] b}-g_{b[c} R_{d] a}-\frac{R}{3} g_{a[c} g_{d] b}
$$

where $R_{a b}$ and $C_{a b c d}$ are the Ricci and Weyl tensors, respectively, and $R \equiv R_{c}^{c}$ is the Ricci scalar. The Einstein equations in the form

$$
R_{a b}=8 \pi G\left(T_{a b}-\frac{1}{2} g_{a b} T\right)
$$

and their contraction $R=-8 \pi G T$, where $T \equiv T^{c}{ }_{c}$, yield (in conjunction with Equation (2))

$$
h^{a c} h^{b d} R_{a b c d}=h^{a c} h^{b d} C_{a b c d}+8 \pi G h^{a c} h^{b d}\left[g_{a[c} T_{d] b}-g_{b[c} T_{d] a}-\frac{T}{2}\left(g_{a[c} g_{d] b}-g_{b[c} g_{d] a}\right)\right]
$$

It is easy to see that

$$
\begin{aligned}
& h^{a c} h^{b d}\left(g_{a[c} T_{d] b}-g_{b[c} T_{d] a}\right)=h^{a b} T_{a b} \\
& h^{a c} h^{b d}\left(g_{a[c} g_{d] b}-g_{b[c} g_{d] a}\right)=2
\end{aligned}
$$

which reduces $M_{\mathrm{H}}$ to the sum of a matter contribution and of a Weyl contribution (A version of Equation (8) for scalar-tensor gravity appears in Reference [42])

$$
M_{\mathrm{H}}=\sqrt{\frac{A}{16 \pi}} \int_{\mathcal{S}} \mu\left(h^{a b} T_{a b}-\frac{2 T}{3}\right)+\frac{1}{8 \pi G} \sqrt{\frac{A}{16 \pi}} \int_{\mathcal{S}} \mu h^{a c} h^{b d} C_{a b c d}
$$


The first integral on the right hand side of Equation (8), which does not contain Newton's constant $G$, is determined directly by the matter present on $\mathcal{S}$ and it vanishes in vacuo. The second integral, which contains $G$ and does not depend on matter directly, can be seen as a "pure field" contribution, although it contains the 2-metric $h_{a b}$ which is also determined by matter through the Einstein equations.

To visualize the first contribution, imagine the special (but very common in the literature) situation in which the matter content of spacetime is a perfect fluid, described by the stress-energy tensor

$$
T_{a b}=(P+\rho) u_{a} u_{b}+P g_{a b}
$$

where $\rho, P$, and $u^{c}$ are the energy density, (isotropic) pressure, and 4-velocity field of the fluid, respectively. If we choose the 2 -surface $\mathcal{S}$ in such a way that the 4 -velocity $u^{a}$ is normal to it, i.e., $h_{a b} u^{b}=0$, then we have

$$
h^{a b} T_{a b}-\frac{2 T}{3}=\frac{2 \rho}{3}
$$

and the quasilocal mass does not depend explicitly on the pressure, a feature which was already noted in References [38,39] in spherical symmetry and is now generalized to arbitrary spacetimes. Of course, realistically, there will be an equation of state of the fluid relating energy density and pressure. Nevertheless, the property that pressures do not contribute to $M_{\mathrm{H}}$ is noteworthy because one of the first things that one learns in relativity is that the pressure of a fluid gravitates together with its energy density, for example in the Tolman-Oppenheimer-Volkoff equation for interior solutions, or in the Einstein-Friedmann equations for Friedmann-Lemaître-Robertson-Walker cosmology [36]. In this sense, it seems that this contribution to the Hawking mass behaves more like a Newtonian than a relativistic mass.

Let us consider now an imperfect fluid, the stress-energy tensor of which has the general form

$$
T_{a b}=\rho u_{a} u_{b}+P \gamma_{a b}+q_{a} u_{b}+q_{b} u_{a}+\Pi_{a b}
$$

where $\gamma_{a b}$ is the 3-metric on the 3-space orthogonal to $u^{a}$ and is defined by $g_{a b}=-u_{a} u_{b}+\gamma_{a b}, q^{a}$ is a purely spatial heat current vector satisfying $q^{c} u_{c}=0$, and $\Pi_{a b}$ is the symmetric and trace-free shear tensor. For such an imperfect fluid the trace is still $T=-\rho+3 P$ and one finds that

$$
h^{a b} T_{a b}-\frac{2 T}{3}=\frac{2}{3} \rho+h^{a b} \Pi_{a b}=\frac{2}{3} \rho+\Pi^{2}{ }_{2}+\Pi^{3}{ }_{3}=\frac{2}{3} \rho-\Pi^{1}{ }_{1}
$$

labeling $\left(x^{2}, x^{3}\right)$ the coordinates on $\mathcal{S}$. Therefore, while the principal stresses associated with directions lying along $\mathcal{S}$ do not contribute to the mass $M_{\mathrm{H}}$, the one corresponding to the third direction normal to $\mathcal{S}$ does contribute to $M_{\mathrm{H}}$ (when it is non-zero) hence, to some extent, non-isotropic stresses gravitate according to Hawking's prescription.

As a special case of an imperfect fluid, bulk and viscous stresses can be introduced as follows:

$$
P=P_{(\mathrm{e})}+P_{(\mathrm{ne})}
$$

where $P_{(\mathrm{e})}$ is an equilibrium pressure and $P_{(\text {ne) }}$ is a non-equilibrium component, while viscosity is described by $P_{(\mathrm{ne})}=-\zeta \theta$, with $\eta$ a viscosity coefficient and $\theta=\nabla^{c} u_{c}$ the expansion of the timelike congruence with tangent $u^{c}$. Although not the most general form of an imperfect fluid, this is in fact the form reported in several textbooks (e.g., $[43,44])$ and technical articles. The stress-energy tensor in this case is

$$
T_{a b}=\rho u_{a} u_{b}+P_{(\mathrm{e})} \gamma_{a b}-\zeta \theta \gamma_{a b}+q_{a} u_{b}+q_{b} u_{a}-2 \eta \sigma_{a b}
$$


which gives

$$
h^{a b} T_{a b}-\frac{2 T}{3}=\frac{2}{3} \rho+2 \eta \sigma_{1}^{1}
$$

\section{3. "Newtonian" Character of the Hawking mass}

We now decompose further the gravitational contribution to the Hawking mass with the purpose of identifying its "Newtonian" and "non-Newtonian" parts relative to an observer with 4-velocity $u^{a}$. To give a meaning to these adjectives, we decompose the Weyl tensor into its electric and magnetic parts. While the electric part $E_{a b}$ of the Weyl tensor has a Newtonian analogue, its magnetic part $H_{a b}$ does not [34] and we identify the "Newtonian" contribution to $M_{\mathrm{H}}$ with the terms due to $E_{a b}$ in the second integral on the right hand side of Equation (8), and the non-Newtonian part with the contribution due to $H_{a b}$.

To proceed, remember that electric and magnetic parts of the Weyl tensor are defined relative to an observer. It is natural to identify the 4-velocity $u^{a}$ of the observer with the timelike unit normal to the spacelike 2-surface $\mathcal{S}$. Then the electric and magnetic parts of the Weyl tensor are (Here we follow the definitions of [35], which differ from that of [34] in the magnetic part of the Weyl tensor and correct a sign error)

$$
\begin{aligned}
& E_{a c}(u)=C_{a b c d} u^{b} u^{d} \\
& H_{a c}(u)=\frac{1}{2} \eta_{a b p q} C_{c e}^{p q} u^{b} u^{e}
\end{aligned}
$$

respectively, where $\eta_{a b c d}=\sqrt{-g} \epsilon_{a b c d}$ with $\epsilon_{a b c d}$ the alternating symbol and $g$ the determinant of the metric tensor $g_{a b}$. In other words, $\eta^{a b c d}=\eta^{[a b c d]}$ and $\eta^{0123}=1 / \sqrt{-g} . E_{a b}$ and $H_{a b}$ are purely spatial, symmetric, and trace-free,

$$
\begin{gathered}
E_{a b} u^{a}=E_{a b} u^{b}=H_{a b} u^{a}=H_{a b} u^{b}=0 \\
E_{a b}=E_{(a b)} \quad H_{a b}=H_{(a b)} \\
E^{a}{ }_{a}=H^{a}{ }_{a}=0
\end{gathered}
$$

The Weyl tensor is reconstructed from its electric and magnetic parts according to [34,35]

$$
C_{a b c d}=\left(g_{a b e f} g_{c d p q}-\eta_{a b e f} \eta_{c d p q}\right) u^{e} u^{p} E^{f q}-\left(\eta_{a b e f} g_{c d p q}+g_{a b e f} \eta_{c d p q}\right) u^{e} u^{p} H^{f q}
$$

where

$$
g_{a b e f} \equiv g_{a e} g_{b f}-g_{a f} g_{b e}
$$

Therefore, we have

$$
\begin{aligned}
C_{a b c d}= & \left(g_{a e} g_{b f}-g_{a f} g_{b e}\right)\left(g_{c p} g_{d q}-g_{c q} g_{d p}\right) u^{e} u^{p} E^{f q}-\eta_{a b e f} \eta_{c d p q} u^{e} u^{p} E^{f q} \\
& -\left[\eta_{a b e f}\left(g_{c p} g_{d q}-g_{c q} g_{d p}\right)+\left(g_{a e} g_{b f}-g_{a f} g_{b e}\right) \eta_{c d p q}\right] u^{e} u^{p} H^{f q} \\
= & u_{a} u_{c} E_{b d}-u_{a} u_{d} E_{b c}-u_{b} u_{c} E_{a d}+u_{b} u_{d} E_{a c}-\eta_{a b e f} \eta_{c d p q} u^{e} u^{p} E^{f q} \\
& -\eta_{a b e f} u_{c} u^{e} H_{d}^{f}+\eta_{a b e f} u^{e} u_{d} H_{c}^{f}-u_{a} u^{p} \eta_{c d p q} H_{b}^{q}+u_{b} u^{p} \eta_{c d p q} H_{a}^{q}
\end{aligned}
$$


By contracting twice with the (inverse) 2-metric $h^{a b}$ most terms vanish, leaving

$$
h^{a c} h^{b d} C_{a b c d}=-\eta_{a b e f} \eta_{c d p q} h^{a c} h^{b d} u^{e} u^{p} E^{f q}
$$

To summarize, after the two splittings are performed, the Hawking mass can be written as

$$
M_{\mathrm{H}}=\sqrt{\frac{A}{16 \pi}} \int_{\mathcal{S}} \mu\left(h^{a b} T_{a b}-\frac{2 T}{3}\right)-\frac{1}{8 \pi G} \sqrt{\frac{A}{16 \pi}} \int_{\mathcal{S}} \mu \eta_{a b e f} \eta_{c d p q} h^{a c} h^{b d} u^{e} u^{p} E^{f q}
$$

The "pure gravity" contribution to $M_{\mathrm{H}}$ comes only from the electric part of the Weyl tensor, which has a counterpart in Newtonian gravity [34]. The magnetic part of $C_{a b c d}$ which, on the contrary, has no Newtonian counterpart [34], gives zero contribution. In this sense, the Hawking mass is "Newtonian". Although our meaning of the adjective "Newtonian" is quite different from that of Reference [23], the spirit is not too different and Equation (25) can be seen as a statement that the Hawking mass is "Newtonian" on the same lines of the result of [23]. The statement is much stronger, in the sense that our discussion leading to Equation (25) is not restricted to spherical symmetry.

\section{Conclusions}

It is intriguing that, in spherical symmetry, the Hawking quasilocal energy (which reduces to the Misner-Sharp-Hernandez one) is found to have "Newtonian" character [23]. A previous discussion of the Newtonian character in the literature [23] relied on the behaviour of timelike geodesics in spherically symmetric general-relativistic spacetimes. When one tries to extend this result to arbitrary general-relativistic spacetimes, which we have done here, one needs to identify what "Newtonian character" means. It is in principle possible that spherical symmetry hides some physical features of the Newtonian or non-Newtonian nature of the quasilocal mass, which would then be brought into light by relaxing this symmetry. While there are a priori several possibilities, it is rather natural to think of characterizing Newtonianity by using the decomposition of the Weyl tensor into its electric and magnetic parts. In fact, the magnetic part of the Weyl tensor $C_{a b c d}$ does not have a Newtonian counterpart, while its electric part does, corresponding to tidal fields [27,34,35]. This approach is quite different from that of Reference [23] and could, in principle, lead to completely different results. The problem is how to relate the Hawking quasilocal mass with the decomposition of $C_{a b c d}$. Fortunately, this question is answered easily by using the contracted Gauss Equation (2). The splitting of the Hawking mass $M_{\mathrm{H}}$ into a matter part and a purely gravitational part (determined by $C_{a b c d}$ ) is then straightforward. When the matter content of spacetime is a perfect fluid or a mixture of perfect fluids, this part of $M_{\mathrm{H}}$ does not depend on the (isotropic) pressure, contributing to the interpretation of $M_{\mathrm{H}}$ as a Newtonian, as opposed to relativistic, quantity. However, for an imperfect fluid $M_{\mathrm{H}}$ depends on the principal stress in the spatial direction orthogonal to the compact spacelike 2-surface $\mathcal{S}$ on which $M_{\mathrm{H}}$ is defined (but not on the principal stresses along the two directions in $\mathcal{S}$ ).

Then, the decomposition of the Weyl tensor into an electric ("Newtonian") part and a magnetic ("non-Newtonian") part determines a corresponding splitting of the Hawking quasilocal mass. However, the magnetic Weyl part vanishes identically in all situations in general relativity, leaving only the electric part that we identified as "Newtonian". This means that the Hawking mass is due only to 
contributions from matter distributions and from tidal fields. Our procedure in identifying Newtonian and non-Newtonian contributions is not directly applicable to other quasilocal energy prescriptions. What is more, the characterization of "Newtonian" followed here may ultimately not be the most convenient one. These issues become more relevant in light of relativistic virial theorems [45] and the application of the quasilocal energy to cosmological perturbations [2,22] and will be considered further in the future.

\section{Acknowledgments}

This work is supported by the Natural Science and Engineering Research Council of Canada and by Bishop's University.

\section{Conflicts of Interest}

The author declares no conflict of interest.

\section{References}

1. Szabados, L.B. Quasi-local energy-momentum and angular momentum in general relativity. Living Rev. Rel. 2009, 12, doi:10.12942/lrr-2009-4.

2. Faraoni, V.; Lapierre-Léonard, M.; Prain, A. Do Newtonian large-scale structure simulations fail to include relativistic effects? Phys. Rev. D 2015, 92, doi:10.1103/PhysRevD.92.023511.

3. Souriau, J.M. Un modèle d'univers confronté aux observations. In Dynamics and Processes, Proceedings of the Third Encounter in Mathematics and Physics, Bielefeld, Germany, 30 November-4 December 1981; Lecture Notes in Mathematics; Blanchard, P., Streit, W., Eds.; Springer-Verlag: Berlin, Germany, 1981; Volume 1031, pp. 114-160.

4. Stuchlik, Z. The motion of test particles in black-hole backgrounds with non-zero cosmological constant. Bull. Astron. Inst. Czechoslov. 1983, 34, 129-149.

5. Stuchlik, Z.; Hledik, S. Some properties of the Schwarzschild-de Sitter and Schwarzschild-anti-de Sitter spacetimes. Phys. Rev. D 1999, 60, doi:10.1103/PhysRevD.60.044006.

6. Stuchlik, Z.; Slany, P.; Hledik, S. Equilibrium configurations of perfect fluid orbiting Schwarzschild-de Sitter black holes. Astron. Astrophys. 2000, 363, 425-439.

7. Stuchlik, Z. Influence of the relict cosmological constant on accretion discs. Mod. Phys. Lett. A 2005, 20, 561-576.

8. Mizony, M.; Lachiéze-Rey, M. Cosmological effects in the local static frame. Astron. Astrophys. 2005, 434, 45-52.

9. Stuchlik, Z.; Schee, J. Influence of the cosmological constant on the motion of Magellanic Clouds in the gravitational field of Milky Way. JCAP 2011, 9, doi:10.1088/1475-7516/2011/09/018.

10. Roupas, Z.; Axenides, M.; Georgiou, G.; Saridakis, E.N. Galaxy clusters and structure formation in quintessence versus phantom dark energy universe. Phys. Rev. D 2014, 89, doi:10.1103/PhysRevD.89.083002.

11. Nolan, B.C. Particle and photon orbits in McVittie spacetimes. Class. Quantum Grav. 2014, 31, doi:10.1088/0264-9381/31/23/235008. 
12. Pavlidou, V.; Tomaras, T.N. Where the world stands still: Turnaround as a strong test of $\Lambda$ CDM cosmology. JCAP 2014, 1409, doi:10.1088/1475-7516/2014/09/020.

13. Pavlidou, V.; Tetradis, N.; Tomaras, T.N. Constraining dark energy through the stability of cosmic structures. JCAP 2014, 1405, doi:10.1088/1475-7516/2014/05/017.

14. Maciel, A.; Le Delliou, M.; Mimoso, J.P. A dual null formalism for the collapse of fluids in a cosmological background. 2015, arXiv:1506.07122 [gr-qc]. arXiv.org e-Print archive. Available online: http://arxiv.org/abs/1506.07122 (accessed on 29 October 2015).

15. Le Delliou, M.; Mimoso, J.P.; Mena, F.C.; Fontanini, M.; Guariento, D.C.; Abdalla, E. Separating expansion and collapse in general fluid models with heat flux. Phys. Rev. D 2013, 88, doi:10.1103/ PhysRevD.88.027301.

16. Mimoso, J.P.; Le Delliou, M.; Mena, F.C. Local conditions separating expansion from collapse in spherically symmetric models with anisotropic pressures. Phys. Rev. D 2013, 88, doi:10.1103/ PhysRevD.88.043501.

17. Mimoso, J.P.; Le Delliou, M.; Mena, F.C. Spherically symmetric models: Separating expansion from contraction in models with anisotropic pressures. AIP Conf. Proc. 2011, 1458, 487-490.

18. Mimoso, J.P.; Le Delliou, M.; Mena, F.C. Separating expansion from contraction in spherically symmetric models with a perfect-fluid: Generalization of the Tolman-Oppenheimer-Volkoff condition and application to models with a cosmological constant. Phys. Rev. D 2010, 81, doi:10.1103/PhysRevD.81.123514.

19. Le Delliou, M.; Mimoso, J.P. Separating expansion from contraction and generalizing TOV condition in spherically symmetric models with pressure. AIP Conf. Proc. 2009, 1122, 316-319.

20. Tanoglidis, D.; Pavlidou, V.; Tomaras, T.N. Statistics of the end of turnaround-scale structure formation in $\Lambda$ CDM cosmology. 2014, arXiv:1412.6671 [astro-ph.CO]. arXiv.org e-Print archive. Available online: http://arxiv.org/abs/1412.6671 (accessed on 29 October 2015).

21. Busha, M.T.; Adams, F.C.; Wechsler, R.H.; Evrard, A.E. Future evolution of structure in an accelerating universe. Astrophys. J. 2003, 596, 713-724.

22. Faraoni, V.; Lapierre-Léonard, M.; Prain, A. Turnaround radius in an accelerated universe with quasi-local mass. JCAP 2015, 10, doi:10.1088/1475-7516/2015/10/013.

23. Blau, M.; Rollier, B. Brown-York energy and radial geodesics. Class. Quantum Grav. 2008, 25, doi:10.1088/0264-9381/25/10/105004.

24. Misner, C.W.; Sharp, D.H. Relativistic equations for adiabatic, spherically symmetric gravitational collapse. Phys. Rev. 1964, 136, B571-B576.

25. Hernandez, W.C.; Misner, C.W. Observer time as a coordinate in relativistic spherical hydrodynamics. Astrophys. J. 1966, 143, 452-464.

26. Brown, J.D.; York, J.W. Quasilocal energy and conserved charges from the gravitational action. Phys. Rev. D 1993, 47, 1407-1419.

27. Matte, A. Sur des nouvelles solutions oscillatoires des equations de la gravitation. Can. J. Math. 1953, 5, 1-16.

28. Matarrese, S.; Pantano, O.; Saez, D. A general relativistic approach to the nonlinear evolution of collisionless matter. Phys. Rev. D 1993, 47, 1311-1323.

29. Bertschinger, E.; Jain, B. Gravitational instability of cold matter. Astrophys. J. 1994, 431, 486-494. 
30. Bertschinger, E.; Hamilton, A.J.S. Lagrangian evolution of the Weyl tensor. Astrophys. J. 1994, 435, 1-7.

31. Bruni, M.; Matarrese, S.; Pantano, O. Dynamics of silent universes. Astrophys. J. 1995, 445, 958-977.

32. Croudace, K.M.; Parry, J.; Salopek, D.S.; Stewart, J.M. Applying the Zeldovich approximation to general relativity. Astrophys. J. 1994, 423, 22-32.

33. Kofman, L.; Pogosyan, D. Dynamics of gravitational instability is nonlocal. Astrophys. J. 1995, 442, 30-38.

34. Ellis, G.F.R. General Relativity and Cosmology. In Proceedings of the International School of Physics E. Fermi, Course XLVII, Varenna, Italy, 1969; Sachs, R.K., Ed; Academic Press: New York, NY, USA, 1971; pp. 104-182.

35. Bertschinger, E. Cosmological dynamics. 1993, [arXiv:astro-ph/9503125]. arXiv.org e-Print archive. Available online: http://arxiv.org/abs/astro-ph/9503125 (accessed on 29 October 2015).

36. Wald, R.M. General Relativity; Chicago University Press: Chicago, IL, USA, 1984.

37. Hawking, S. Gravitational radiation in an expanding universe. J. Math. Phys. 1968, 9, 598-604.

38. Hayward, S.A. Quasilocal gravitational energy. Phys. Rev. D 1994, 49, 831-839.

39. Hayward, S.A. Gravitational energy in spherical symmetry. Phys. Rev. D 1996, 53, 1938-1949.

40. Kodama, H. Conserved energy flux from the spherically symmetric system and the back reaction problem in the black hole evaporation. Progr. Theor. Phys. 1980, 63, 1217-1228.

41. Abreu, G.; Visser, M. Kodama time: Geometrically preferred foliations of spherically symmetric spacetimes. Phys. Rev. D 2010, 82, doi:10.1103/PhysRevD.82.044027.

42. Faraoni, V. Quasilocal energy in modified gravity. 2015, arXiv:1508.06845. arXiv.org e-Print archive. Available online: http://arxiv.org/abs/1508.06845 (accessed on 29 October 2015).

43. Misner, C.W.; Thorne, K.S.; Wheeler, J.A. Gravitation; Freeman: San Francisco, CA, USA, 1973.

44. Krasiński, A. Inhomogeneous Cosmological Models; Cambridge University Press: Cambridge, UK, 1997.

45. Uzun, N.; Wiltshire, D.L. Quasilocal energy and thermodynamic equilibrium conditions. Class. Quantum Grav. 2015, 32, doi:10.1088/0264-9381/32/16/165011.

(c) 2015 by the author; licensee MDPI, Basel, Switzerland. This article is an open access article distributed under the terms and conditions of the Creative Commons Attribution license (http://creativecommons.org/licenses/by/4.0/). 